\title{
Inner core rotation from geomagnetic westward drift and a stationary spherical vortex in Earth's core
}

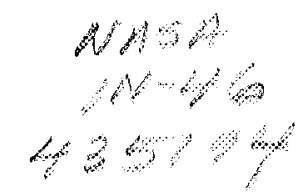

Coerte V. Voorhies, Geodynamics Branch, Code 921 , NASA's Goddard Space Flight Center, Greenbelt, MD 20771

\author{
Phone (301) 286-3530ＦAX (301) 286-1616ｅmail: voorhies@geomag.gsfc.nasa.gov \\ Submitted to Physics of the Earth and Planetary Interiors 31 July, 1998; in revised form 25 November, 1998.
}

\begin{abstract}
The idea that geomagnetic westward drift indicates convective leveling of the planetary momentum gradient within Earth's core is pursued in search of a differentially rotating mean state, upon which various oscillations and secular effects might be superimposed. The desired state conforms to roughly spherical boundary conditions, minimizes dissipative interference with convective cooling in the bulk of the core, yet may aid core cooling by depositing heat in the uppermost core and lower mantle. The variational calculus of stationary dissipation applied to a spherical vortex within the core yields an interesting differential rotation profile, akin to spherical Couette flow bounded by thin Hartmann layers. Four boundary conditions are required. To concentrate shear induced dissipation near the core-mantle boundary, these are taken to be: (i) no-slip at the core-mantle interface; (ii) geomagnetically estimated bulk westward flow at the base of the core-mantle boundary layer; (iii) noslip at the inner-outer core interface; and, to describe magnetic locking of the inner core to the deep outer core, (iv) hydrodynamically stress-free at the inner-outer core boundary. By boldly assuming the axial core angular momentum anomaly to be zero, the super-rotation of the inner core relative to the mantle is calculated to be at most $1.5 \%$ yr.
\end{abstract}

\section{Introduction}

The westward drift of certain features in the geomagnetic field across Earth's surface at a few tenths of a degree per year has long been used to suggest westward drift of Earth's magnetized core (Halley, 1692). To the extent that the field is frozen into an electrically conducting fluid outer core, westward drift indicates westward flow near the top of the core. Bulk westward flow at about $0.12 \%$ yr emerges from somewhat more detailed inversions of secular geomagnetic change (see, e.g., Voorhies, 1995); however, this rate can be reduced by diffusion, hence slipping, of the field through the imperfectly conducting fluid (see, e.g., Voorhies, 1993). Such westward flow poses an interesting problem for those who suspect that, during the course of geologic time, Earth's core and mantle have relaxed to a state wherein the angular momentum of the core is quite close to that of a rigid body rotating with the 
angular velocity of the mantle. (This apart from differential spin up due to inner core solidification, differential tidal spin down, differential precession, and various oscillations).

A slight convective leveling of the planetary azimuthal momentum gradient still seems to offer the most facile explanation of westward drift. Compared with a non-convecting, uniformly rotating state, buoyant, low azimuthal momentum fluid at depth floats towards the upper surface and drifts west; dense, high azimuthal momentum fluid above sinks towards the lower surface and drifts east; and the azimuthal momentum gradient is reduced. The reduction is arguably very small for several reasons: the convective motions are feeble, strongly constrained by the rotation itself, and may be largely confined to spirals on surfaces of constant planetary momentum. Slight departures from motion on such surfaces may nonetheless be realized, notably if they increase convective efficiency by decreasing dissipation or, perhaps for thermo-compositional magneto-convection, by distributing Ohmic and viscous dissipation so as to aid core cooling. Westward drift has thus long provided a reason to consider seriously the convective outer core geodynamo hypothesis, whereby convective fluid motions maintain the aperiodically reversing geomagnetic main field against magnetic diffusion and Ohmic dissipation.

This facile explanation of westward drift clearly requires eastward flow of the fluid at depth in the outer core. If the total angular momentum of the core is quite close to that of a rigid body of identical inertial moment rotating with the mantle, then the speed of the eastward flow at depth will exceed that of the westward flow above so long as the geometric effects of reduced moment arm and volume at depth dominate the increased density of quasihydrostatic compression.

The foregoing argument for eastward flow at depth has long been obvious to students of the secular variation. It is now clear that tight magnetic coupling of eastward flow near the base of the outer core to the similarly high conductivity solid inner core would tend to spin up the inner core. Indeed, deviation from co-rotation shears the magnetic field lines that thread the inner core boundary and are thus embedded in both liquid and solid conductors. Such shear induces electric current and restoring Lorentz torques that tend to return the system towards a magnetically locked equilibrium - perhaps after several resistively damped oscillations (Gubbins, 1981). The essential geophysics was elucidated by Glatzmaier \& Roberts (1995a, b; 1996), via numerical simulation of the core geodynamo, and led to their celebrated prediction of an eastwardly drifting inner core.

Seismologic substantiation of this prediction by Song \& Richards (1996) and Su et al. (1996) suggests considerable uncertainty as to the amplitude of eastward inner core drift. The former obtain about $1.1 \% \mathrm{yr}$; the latter 
obtain about $3 \% \mathrm{yr}(2.27 \pm 0.90 \% \mathrm{yr}, 3.02 \pm 0.42 \% \mathrm{yr}$, and $3.28 \% \mathrm{yr}$ depending upon details of the inversion). Creager (1997) obtains a lower rate of about $0.25^{\circ} / \mathrm{yr}$, while Souriau et al. $(1997 ; 1998)$ question the seismologic detection of inner core rotation itself. This paper shows how eastward drift of a solid inner core can be simply, albeit not unambiguously, calculated from geomagnetic westward drift without recourse to either numerical simulation or models of inner core acoustic anisotropy.

\section{Simple Model and Example}

Consider a planet rotating with sidereal angular velocity $\Omega(r, t)$ and mass density equal to marginally stable reference density $\rho(\mathbf{r})$ plus small perturbation $\delta \rho(r, t)(|\delta \rho / \rho| \ll 1)$. For simplicity, position $\mathbf{r}$ is measured in centered spherical polar coordinates (radius $r$, colatitude $\theta$, and east longitude $\phi$ ) rotating with a rigid mantle at uniform angular velocity $\Omega_{\mathrm{o}} \hat{\mathrm{z}}$ ( $\hat{\mathrm{z}} \equiv \hat{\mathrm{r}} \cos \theta-\theta \mathrm{r} \sin \theta$ ). This filters out secular tidal despin, polar wander, precession, nutation, and decadal and higher frequency fluctutations in the angular velocity of the mantling solid, which fall outside the focus of this paper.

The planetary rotational velocity $\Omega_{0} \hat{z} \times \mathbf{r}$ is eastward $\Omega_{0}{ }_{0} \sin \theta \hat{\phi}$. The planetary momentum density $\mathbf{M}$ is eastward $\mathrm{M}_{\phi}$, is $\hat{\phi} \rho \Omega_{0} r \sin \theta$, and is the planetary azimuthal momentum of a material parcel of unit volume. Clearly $M_{\phi}$ tends to increase with distance away from the rotation axis, $s \equiv$ rsin $\theta$. For a small planetoid of homogeneous mass density $\rho_{0}$, the planetary momentum gradient $\nabla M_{\phi}$ is uniformly steep $\rho_{0} \Omega_{0} \hat{s}$. For a self-gravitating compressible planet,

$$
\nabla M_{\phi}=\rho \Omega_{0}(\hat{s}+s \nabla \rho / \rho)
$$

If the rotation is not too fast, then a roughly spheroidal planet remains nearly spherical, the stratification is nearly radial $\rho(\mathrm{r}), \nabla \rho \approx \hat{\mathrm{r}}_{\mathrm{r}} \rho$, and $\partial_{\mathrm{r}} \rho<0$. If the density scale height is not too small $\left(\rho /\left.\partial_{\mathrm{r}} \rho\right|^{-1}>s\right)$, then $\nabla M_{\phi}$ is not vastly different than for homogeneous density; however, surfaces of constant planetary momentum flare away from the rotation axis as distance from the equatorial plane $|z|$ increases. Such surfaces are more akin to hyperboloids of revolution than right circular cylinders. For terrestrial example, calculated from the seismologic density model of Kennet et al. (1995), the constant $M_{\phi}$ surface tangent to the equator of Earth's solid inner core intercepts the fluid outer core-mantle boundary at colatitude $25.4^{\circ}$ - some $300 \mathrm{~km}$ south of the right circular tangent cylinder intercept at $20.5^{\circ}$. 
To avoid convective leveling of the planetary momentum gradient in a thick, rapidly rotating, roughly spherical annulus such as Earth's electrically conducting liquid outer core, one need only arrange (a) perfect exchange of planetary momentum between rising and falling fluid; (b) perfect confinement of convective motions to surfaces of constant planetary momentum; or (c) perfect exchange of planetary momentum between fluid parcels -moving onto and parcels moving away from each surface of constant planetary momentum. Although case (c) is thought to be an excellent approximation, it seems unlikely that any of these conditions could be perfectly satisfied in a real planet.

Core Angular Momentum Anomaly. The angular momentum density in the core is the sum of planetary and deviatoric portions. The planetary angular momentum density is northward $\left(\mathbf{r} \times \hat{\phi} M_{\phi}=-\theta \rho \Omega_{0} r^{2} \sin \theta\right)$. The deviatoric angular momentum density is due to perturbation density and relative velocity $v(r, t)$. The total angular momentum of the core is the volume integral

$$
\begin{aligned}
\mathbf{L} & =\iiint_{C V}\left[\mathbf{r} \times \hat{\phi} \rho \Omega_{0} r \sin \theta+\mathbf{r} \times\left(\rho \mathbf{v}+\delta \rho \mathbf{v}+\hat{\phi} \delta \rho \Omega_{0} r \sin \theta\right)\right] d V \\
& \equiv \mathbf{L}_{\mathbf{p}}+\Delta \mathbf{L}
\end{aligned}
$$

where $\mathrm{CV}$ denotes core volume. If $\rho(\mathbf{r})$ is axisymmetric $\rho(r, \theta)$, then the planetary angular momentum of the core $\mathbf{L}_{\mathbf{p}}$ is parallel to the reference rotation axis. The deviatoric angular momentum,

$$
\begin{aligned}
\Delta \mathbf{L} & \equiv \iiint_{C V} \mathbf{r} \times\left(\rho \mathbf{v}+\delta \rho \mathbf{v}+\hat{\phi} \delta \rho \Omega_{0} r \sin \theta\right) d V \\
& \approx \iiint_{C V} \mathbf{r} \times\left(\rho \mathbf{v}+\hat{\phi} \delta \rho \Omega_{0} r \sin \theta\right) d V
\end{aligned}
$$

is just the core angular momentum anomaly which some think small $\left(|\Delta \mathbf{L}| \ll\left|\mathbf{L}_{\mathbf{p}}\right|\right)$.

Does the Taylor-Proudman Theorem Inhibit Leveling? To find out, partition the mass transport equation into main $(\nabla \bullet \rho \mathbf{v}=0)$ and fluctuating parts. The curl of an approximate balance between the Coriolis force density $\left(2 \Omega_{\mathrm{o}} \hat{\mathrm{Z}} \times \rho \mathbf{v}\right)$ and hydrostatically uncompensated scaloidal force densities (such as perturbation pressure) gives the Proudman-Taylor constraint $2 \Omega_{\mathrm{o}}(\hat{Z} \bullet \nabla) \rho v \approx 0$ (see, e.g., Gubbins \& Roberts, 1987). The relative momentum density $\rho v$ does not vary much along the direction of the rotation axis; yet motions orthogonal to the rotation axis are allowed and might slightly level $\nabla \mathrm{M}_{\phi}$. Such leveling would appear as a non-zero mean gradient in the deviatoric azimuthal momentum $\left(\rho v_{\phi}+\delta \rho v_{\phi}+\delta \rho \Omega_{0} r \sin \theta\right)$ rather than a change in $\nabla M_{\phi}$ itself. 
Purely azimuthal flow $v_{\phi}(r)$ satisfies the constraint when relative momentum $\rho v_{\phi}$ varies only with $s$ (i.e., $v_{\phi}(r) \approx[\rho(r)]^{-1}\left[f(r \sin \theta)+K_{o}\right]$ where $K_{o}$ is a constant and $f$ is a function of $s$ alone). At fixed $s$, the decrease in $\rho$ with radius, hence distance $|\mathrm{z}|$ from the equatorial plane, can be compensated by faster flow. Surfaces of constant $v_{\phi}$ thus tend to curve towards the rotation axis as $|\mathrm{z}|$ increases. Such curvature resembles a spherical vortex modulating azimuthal flow that would otherwise be invariant along more familiar co-axial right circular cylinders. The effect could be quite large for motions spanning multiple density scale heights, as in a stellar or giant planetary convection zone, but the mass density contrast across Earth's outer core is only $22 \%$. The notion of a spherical vortex persists because Taylor-Proudman conditions are not fully met by magneto-convection of a viscous fluid; because smaller scale motions might excite and maintain such a vortex; because of the shape of the bounding solids; and because of geomagnetic westward drift.

\subsection{Simple Spherical Vortex and Boundary Conditions}

As in many models, inner-outer core and core-mantle transition zones are here approximated by sharp material interfaces. The solid inner core is approximated by a rigid ball of radius $d$, the fluid outer core by a spherical annulus of outer radius $c$ and inner radius $d$, and the reference density by laterally homogeneous $\rho(r)$. For the bulk eastward or westward flows considered here, spherical shells rotate as if rigid bodies; therefore, attention is directed towards the spherical vortex

$$
V(r)=w_{0}(r) \sin \theta \hat{\phi}=\omega(r) r \sin \theta \hat{\phi},
$$

where $w_{0}(r)$ is the equatorial amplitude of bulk eastward flow, $\omega(r)$ is the perturbation angular velocity or "eastward drift", and the relative vorticity is $2 \omega(r) \hat{z}$. Spherical vortex (3a) is partitioned into frozen field and diffusive portions

$$
\omega(r)=\omega_{B}(r)+\omega_{\eta}(r)
$$

where $\omega_{B}$ represents geomagnetic drift in the frozen-flux approximation and $\omega_{\eta}(r)$ denotes diffusive slip. The radially varying ratio $\left|\omega / \omega_{\eta}\right|$ indicates the relative importance of advection to diffusion in the vortex.

The hypothetical kinematic model (3a) is not intended to satisfy conditions needed for the Taylor-Proudman theorem, the rigidized sphere model of differential precession (Vanyo, 1984), a dynamical simulation (Glatzmaier \& Roberts, 1995a, b; 1996), or a thermal wind model (Aurnou et al., 1996; 1998). The rigidized sphere model would correspond to a homogeneous spherical vortex beneath a boundary layer $\left(\omega\left(r \leq c^{-}\right) \approx 0.1 \% \mathrm{yr}\right)$. Whether or not an inhomogeneous spherical vortex is excited and maintained by smaller scale, possibly turbulent, convective and/or 
wave motions $(\mathbf{u}=\mathbf{v}-\mathbf{v})$, it conforms to the boundaries more naturally than a cylindrical vortex and thus eases discussion of boundary conditions.

Although the outer core might slip past the mantle almost as easily as mercury under leaded glass, radius $\mathrm{c}^{-}$is used to denote the base of a thin viscous boundary layer separating a free-streaming fluid core from a rigid mantle. At the edge of the free-stream,

$$
\begin{aligned}
& \omega\left(c^{-}\right)=\omega_{B}\left(c^{-}\right)+\omega_{\eta}\left(c^{-}\right) \\
& \omega_{B}\left(c^{-}\right)=-0.1157^{\circ} / y r
\end{aligned}
$$

The illustrative numerical value is the 1945-1980 mean from the preferred solution of Voorhies (1995; the value $-0.121 \%$ yr was for 1967.5$)$. Inversions for steady flow and steady flux diffusion suggest some eastward diffusive slip $0.5\left|\omega_{B}\left(c^{-}\right)\right| \geq \omega_{\eta}\left(c^{-}\right) \geq 0$; steady, surfically geostrophic flow inversions suggest a westward diffusive slip $\omega_{\eta}\left(c^{-}\right) \leq 0$ and slower westward magnetic drift (Voorhies, 1993). The maximum westward flow used below is the frozen-flux extreme, $-\omega\left(c^{-}\right)=-\omega_{B}\left(c^{-}\right)$.

For a rigid mantle, the hydrodynamic no-slip core-mantle interface condition is

$$
\omega(r \geq c)=0 .
$$

For a rigid inner core, the hydrodynamic no-slip inner core boundary condition is

$$
\omega(\mathrm{r} \leq \mathrm{d})=\omega(\mathrm{d})=\lim _{\delta \rightarrow 0^{+}} \omega(\mathrm{d}+\delta)
$$

Viscous and magnetic locking of a rigid inner core to fluid at and near the base of the outer core suggest little or no slip, and little or no shear, at the inner boundary. At the desired equilibrium, neither advection nor shear of magnetic field lines threading the inner core excite restoring Lorentz torques. The no shear condition amounts to the hydrodynamic stress-free inner boundary condition,

$$
\omega^{\prime}(\mathrm{d})=0
$$

where $\omega^{\prime}$ denotes $\partial_{\mathrm{r}} \omega$. The combined no-slip/no-stress conditions yield $\omega\left(d^{-}\right)=\omega(d)=\omega\left(d^{+}\right)$. The intent of $(3 f)$ is to filter out, not rule-out, oscillations about, and secular trends in, the desired equilibrium. Granting conditions (3cf), diffusively uncompensated westward drift $\omega\left(c^{-}\right) \approx \omega_{B}\left(c^{-}\right)$offers kinematic driving for a non-trivial spherical vortex. Gravitational locking of the inner core to the mantle (Buffet, 1997) would further require $\omega(\mathrm{d})$ to be zero. 


\subsection{Axial Angular Momentum Anomaly for Small Density Perturbations}

For spherical vortex (3a), the integrand in equation (2c) for $\Delta L_{z}$ would vanish everywhere if $\delta \rho / \rho$ were equal to $-\omega(\mathrm{r}) / \Omega_{\mathrm{o}}$. In the frozen-flux limit of (3c), such detailed balance would have $\delta \rho\left(c^{-}\right) / \rho\left(c^{-}\right)$be $8.8 \times 10^{-7}$. Provided the volume integral of $\delta \rho$ vanishes, this density surplus above would imply a density deficit, hence eastward flow, below. For example, if surplus mass in the shell of radius $\mathrm{c}^{-}$and thickness $\delta \mathrm{r}$ were balanced by a mass deficit in an equally thin shell of radius $d^{+}$, then $\delta \rho\left(d^{+}\right)$would be $-(c / d)^{2} \delta \rho\left(c^{-}\right)$, or $-8.2 \delta \rho\left(c^{-}\right)$; moreover, with $\varepsilon \equiv d / c$,

$$
\omega\left(d^{+}\right)=-\Omega_{0} \delta \rho\left(d^{+}\right) / \rho\left(d^{+}\right)=\Omega_{0}[c / d]^{-2} \delta \rho\left(c^{-}\right) / \rho\left(d^{+}\right)=-\omega\left(c^{-}\right) \varepsilon^{2} \rho\left(c^{-}\right) / \rho\left(d^{+}\right)
$$

would be $-6.67 \omega\left(c^{-}\right)$(using $\varepsilon=0.3499$ and $\rho_{o}\left(c^{-}\right) / \rho_{o}\left(d^{+}\right)=0.8168$ from Kennet et al., 1995). The corresponding eastward drift of a magnetically locked inner core would be about $0.8 \%$ r.

If buoyancy and Coriolis force densities are the same order of magnitude in a convecting outer core, then $\delta \rho / \rho$ $\approx\left|2 \Omega_{\mathrm{o}} \times \mathrm{v} / \mathrm{g}\right| \approx 4 \times 10^{-9}$. The density perturbation needed for detailed balance, being up to 200 times this value, seems quite unstable. Perturbation angular momentum density shall thus be omitted compared with relative angular momentum density $(r \times \rho v)$. The axial angular momentum anomaly $(2 c)$ for spherical vortex ( $3 a)$ thus reduces to

$$
\begin{aligned}
\Delta L_{z} & =\int_{0}^{2 \pi} \int_{0}^{\pi} \int_{0}^{c} r \sin \theta[\rho(r) w(r) \sin \theta] r^{2} \sin \theta d r d \theta d \phi \\
& =(8 \pi / 3) \int_{0}^{c} \rho(r) \omega(r) r^{4} d r .
\end{aligned}
$$

Although $\rho(r)$ has long been inferred seismologically with great confidence, $\omega(r)$ is needed to evaluate $\Delta \mathrm{L}_{\mathrm{Z}}$. From (3c), the key supposition $\Delta \mathrm{L}_{\mathrm{z}}=0$, and several arbitrary differential rotation profiles and boundary conditions, I calculated inner core drifts ranging from $0.2 \% \mathrm{yr}$ to $3 \%$. The agreement with the range of seismologic estimates appears wholly fortuitous. The following sample reveals the ambiguities and shortcomings of such ad hoc calculations, yet suggests a more satisfactory approach.

\subsection{A Parabolic Shot at Inner Core Rotation}

If $\Delta \mathrm{L}_{\mathrm{Z}}$ from (5b) were steady as well as small, then the hydrodynamic stress-free condition at the base of the viscous sub-layer,

$$
\left.\mathrm{r} \partial_{\mathrm{r}}\left(\mathrm{r}^{-1} \mathrm{~V}_{\phi}\right)\right|_{\mathrm{c}^{-}}=\mathrm{rsin} \theta \omega^{\prime}\left(\mathrm{c}^{-}\right)=0
$$


seems a fair substitute for (3d) because it filters out viscous exchange of angular momentum between the mantle and the main-stream. Equations (6a) and (3c) together give a free-slip main-stream boundary condition.

Of the infinity of profiles that satisfy (6a), (3c) and (3e), consider the simple quadratic form

$$
\omega\left(d \leq r \leq c^{-}\right)=\omega\left(c^{-}\right)-\left(\frac{c-r}{c-d}\right)^{2}\left[\omega\left(c^{-}\right)-\omega(d)\right] .
$$

Vastly more complicated forms for $\omega(\mathrm{r})$ with much more shear and curvature tend to increase viscous dissipation and diffuse away more rapidly. By the well-known omega-effect, they also induce magnetic fields with much more shear and curvature which, in turn, tend to increase Ohmic dissipation and diffuse away more rapidly. Such complicated, seemingly transient, forms for $\omega(r)$ are not selected because the excess dissipation in the body of the outer core interferes with the task at hand: cooling the core over geologic time by smaller scale thermocompositional convective motions. (This is formalized in section 3).

For uniform density throughout the core, substitution of (6b) into (5b) and setting the resulting integral for $\Delta \mathrm{L}_{\mathrm{Z}}$ to zero yields, after some algebra,

$$
\omega(d)=-\omega(c)\left[\frac{1-\varepsilon^{5}-Q^{*}}{\varepsilon^{5}+Q^{*}}\right],
$$

where $\varepsilon \equiv(d / c)=0.3499$ as before and

$$
\left.Q^{*} \equiv(1-\varepsilon)^{2}\right)\left[1 / 21-\varepsilon^{5}+5 \varepsilon^{6} / 3-5 \varepsilon^{7} / 7\right)=0.1064
$$

The eastward drift of the inner core from $(7 a, b)$ is

$$
\omega\left(\mathrm{r} \leq \mathrm{d}^{+}\right)=-7.96 \omega\left(\mathrm{c}^{-}\right)=0.92 \% \mathrm{yr}
$$

where the last step sets $\omega\left(c^{-}\right)$to $\omega_{B}\left(c^{-}\right)$above. In this example, the solid inner core reduces the jump in $\omega$ across the outer core; indeed, if $\varepsilon$ were zero, then $\omega(0)$ would be $-20 \omega\left(c^{-}\right)$.

When uniform core density is replaced with bilinear density variation across the outer core and across the inner core, equation (6b) and the assumption of zero $\Delta \mathrm{L}_{\mathrm{z}}$ yield a modified version of $(7 \mathrm{a}-\mathrm{c})$ that reduces to

$$
\omega\left(\mathrm{r} \leq \mathrm{d}^{+}\right)=-7.383 \omega\left(\mathrm{c}^{-}\right)=0.85 \% \mathrm{yr}
$$

for the boundary densities of Kennet et al. (1995). Increased density at depth decreases the eastward flow required to null the axial core angular momentum anomaly, by about $10 \%$ in this example. 
Differential rotation profile (6b) is not only arbitrary, it does not quite satisfy (3f). The shear at the inner boundary proves quite small, but the accompanying dissipation would not seem to aid inner core solidification and core cooling. In contrast, the concentration of shear induced dissipation into a thin upper boundary layer may aid core cooling by steepening the temperature gradient across the core-mantle interface; this speeds conductive heat transport into, and perhaps helps drive convective heat transport across, the deep mantle. Core motions that pump magnetic energy into the field outside the core and drive Ohmic dissipation in the mantle also export energy from an overheated core, presumably a core that cools, contracts and liberates gravitational energy in accord with Hamilton's, if not Fermat's, principle.

\section{Variational Method}

If a particular core angular momentum anomaly is stationary against perturbations in its underlying differential rotation, then the planet might settle into small oscillations about this state. Such a state could be energetically accessible if it does not cause too much dissipation $D$ of magnetic and kinetic energy. One might thus seek a state wherein (i) $\Delta \mathbf{L}$ is stationary against perturbations in $\omega$; (ii) there is no angular momentum exchange between the outer core and its bounding solids; and (iii) the dissipation in the core is stationary and minimum. This nonmagnetic, uniformly rotating state has evidently not yet been attained, perhaps due to thermo-compositional convective core cooling. A non-zero differential rotation excited and maintained by smaller scale convective motions ought not interfere with such cooling by excesssive dissipation in the body of the core; however, a concentration of dissipation by the differential rotation into a thin core-mantle boundary layer and in the overlying mantle may aide core cooling.

The importance attached to conditions (i), (ii), and (iii) above is thus reversed. The desired differential rotation profile has stationary dissipation, preferably concentrated near the outer boundary via conditions (3c, d). Angular momentum exchange is discouraged via inner-outer core locking conditions $(3 \mathrm{e}, \mathrm{f})$; the resistive mantle is left unlocked, not only to increase dissipation near the core-mantle boundary, but because core viscous torque on the mantle turns out to be feeble and can be compensated by magnetic or topographic, if not gravitational, torque (see, e.g., Voorhies, 1991). With viscous boundary layers resolved, condition (6a) is not needed.

Stationarity of $\mathrm{D}(\omega)+\lambda \Delta \mathrm{L}_{\mathrm{z}}(\omega)$ is written

$\delta\left\{\mathrm{D}+\lambda \Delta \mathrm{L}_{\mathrm{z}}\right\}=0$, 
where $\delta$ denotes perturbations in differential rotation profile and $\lambda$ is a Lagrange multipler. Only magnetic and viscous dissipation directly attributed to spherical vortex (3a) alone are included here. The stationary dissipation solution described below sets $\lambda=0$; however, some interesting side cases use non-zero $\lambda$.

\subsection{Magnetic and Viscous Dissipation}

For a Newtonian fluid with rate of strain $e_{i j}$, molecular kinematic shear viscosity $v$, and viscous stress $p_{i j}$ equal to $2 \rho v \mathrm{e}_{\mathrm{ij}}+2 \rho v \delta_{\mathrm{ij}} \mathrm{e}_{\mathrm{kk}} / 3$, the viscous dissipation for solenoidal flow (3a) alone is

$$
\begin{aligned}
D_{V}(\omega) & =\iiint_{C V} 2 \rho v\left(e_{r \phi}{ }^{2}+e_{\phi r}^{2}\right) d V \\
& =\iiint_{C V} \rho v\left(\partial_{r}(\omega)^{2} r^{2} \sin ^{2} \theta d V\right. \\
& =(8 \pi / 3) \int_{0}^{c} \rho v r^{4}\left(\omega^{\prime}\right)^{2} d r
\end{aligned}
$$

(see, e.g., Chandrasekahr, 1961). For a rigidly rotating inner core, the lower limit of integration in (9a) can be reset to $\mathrm{d}$. Viscous dissipation in a high viscosity inner core can remain much less than in the outer core, provided the magnitude of viscous stress is similar in both regions.

Ohmic dissipation of Amperian current (density $\mathbf{J}$ ) in the liquid metal core of electric conductivity $\sigma$

$$
D_{\sigma}=\int \sigma^{-1} J^{2} d V
$$

is thought to dominate viscous dissipation. By Ohm's law for conductors moving in arbitrary magnetic field B,

$$
J=\sigma(\mathbf{E}+\mathbf{v} \times \mathbf{B})
$$

an electromotive force $\mathbf{v} \times \mathbf{B}$ equal and opposite to electric field $\mathbf{E}$ causes no current and no Ohmic dissipation. Motions inferred in the frozen-flux approximation, wherein $\mathbf{E}+v \times \mathbf{B}$ is set to zero to ensure finite $\mathbf{J}$ despite large $\sigma$, therefore cause no Ohmic dissipation.

We choose the solenoidal vector potential $\mathbf{A}$ appropriate to the Coulomb gauge $\left(\mathbf{B}=\nabla \times \mathbf{A}, \mathbf{E}=-\nabla \Phi-\partial_{\mathrm{t}} \mathbf{A}\right)$. To isolate current due to spherical vortex (3a) alone, denoted $J(\omega)$, the portion of $V(\omega) \times B$ equal to $\partial_{t} A(\omega)$ plus electrostatic $\nabla \Phi(\omega)$ must be removed. Ohm's law for the spherical vortex is thus written

$$
\begin{aligned}
& \mathrm{J}_{\mathrm{r}}(\omega)=-\sigma\left[\omega \mathrm{r} \sin \theta \mathrm{B}_{\theta}+\partial_{\mathrm{r}} \Phi(\omega)+\partial_{\mathrm{t}} \mathrm{A}_{\mathrm{r}}(\omega)\right] \\
& \mathrm{J}_{\theta}(\omega)=\sigma\left[\omega \mathrm{r} \sin \theta \mathrm{B}_{\mathrm{r}}-\mathrm{r}^{-1} \partial_{\theta} \Phi(\omega)-\partial_{\mathrm{t}} \mathrm{A}_{\theta}(\omega)\right]
\end{aligned}
$$


Faraday induction $\left(\partial_{t} B=\nabla \times \partial_{t} A\right)$ due to frozen-flux geomagnetic drift $\omega_{B}$ alone vanishes in a magnetic reference frame rotating at sidereal angular velocity $\Omega_{0}+\omega_{B}$. In such a frame, the relative angular velocity of the fluid is $\omega_{\eta}$ by (3b); therefore, the $\partial_{t} A(\omega)$ term in $(10 b, c)$ is eliminated by replacing $\omega$ with $\omega_{\eta}$

$$
\begin{aligned}
& \mathrm{J}_{\mathrm{r}}(\omega)=-\sigma\left[\omega_{\eta} \mathrm{r} \sin \theta \mathrm{B}_{\theta}+\partial_{\mathrm{r}} \Phi(\omega)\right] \\
& \mathrm{J}_{\theta}(\omega)=\sigma\left[\omega_{\eta} \mathrm{r} \sin \theta \mathrm{B}_{\mathrm{r}}-\mathrm{r}^{-1} \partial_{\theta} \Phi(\omega)\right] .
\end{aligned}
$$

The electrostatic term in (10d, e) must also differentially rotate out, but with a conceivably different Ohmically non-dissipative vortex denoted $\omega^{*}(\mathbf{r})$. Clearly $\mathrm{J}\left(\omega^{*}\right)$ must be zero, as must $\partial_{\mathbf{r}} \mathrm{rJ}_{\theta}\left(\omega^{*}\right)-\partial_{\theta} \mathrm{J}_{\mathrm{r}}\left(\omega^{*}\right)$. For uniform $\sigma$, the latter condition applied to (10d, e) gives

$$
\sigma\left[\partial_{\mathrm{r}}\left(\omega^{*} \mathrm{r}^{2} \sin \theta \mathrm{B}_{\mathrm{r}}\right)+\partial_{\theta}\left(\omega^{*} \mathrm{r} \sin \theta \mathrm{B}_{\theta}\right)\right]=0,
$$

or, for solenoidal $\mathbf{B}$,

$$
\sigma\left[\left(\omega^{*}\right)^{\prime} r^{2} \sin \theta \mathrm{B}_{\mathrm{r}}-\omega^{*} \mathrm{r} \partial_{\phi} \mathrm{B}_{\phi}\right]=0 .
$$

For $\omega^{*}$ to be non-zero, shearing of the field must balance advection of the non-axisymetric field.

A magnetically dissipative vortex $\omega_{\eta}-\omega^{*}$ violates (11), generates non-zero meridional currents $(10)$, and thus induces azimuthal field from meridional field (the famous omega-effect). Substitution of (10d, e), after removing non-dissipative $\omega^{*}$, into $(9 b)$ yields

$$
\mathrm{D}_{\sigma}(\omega)=\iint_{C V} \sigma\left(\omega_{\eta}-\omega^{*}\right)^{2} \mathrm{r}^{2} \sin ^{2} \theta\left(\mathrm{B}_{\mathrm{r}}^{2}+\mathrm{B}_{\theta}^{2}\right) \mathrm{dV}
$$

The definition of weighted mean meridional magnetic pressure,

$$
P_{m}(r) \equiv(3 / 8 \pi) \int_{0}^{2 \pi} \int_{0}^{\pi}(2 \mu)^{-1}\left(B_{r}^{2}+B_{\theta}^{2}\right) \sin ^{3} \theta d \theta d \phi,
$$

and (3b) allow (12a) to be rewritten as

$$
D_{\sigma}(\omega)=(8 \pi / 3) \int_{0}^{c} 2 P_{m} \eta^{-1}\left(\omega-\omega_{B}-\omega^{*}\right)^{2} r^{4} d r
$$

where $\eta$ is magnetic diffusivity $(\mu \sigma)^{-1}$. If $\omega$ equals $\omega_{B}+\omega^{*}$ in a magnetically locked inner core, the lower limit of integration in (12c) can be reset to d. Elsewhere, such balance minimizes Ohmic dissipation due to the vortex alone. Note (12c) excludes the inner product of other currents within the core, notably sources of $P_{m}$, with $\mathbf{J}(\omega)$. 
When $\omega^{*}$ on the left of $(11 \mathrm{~b})$ is replaced with magnetically dissipative $\omega_{\eta}-\omega^{*}$, the resulting term is not generally zero. A non-zero integral of the square of this term is thus a sure sign of, if not directly proportional to, Ohmic dissipation. The imbalance between shear and advection of the magnetic field by a dissipative vortex suggests a possible alternative expression for Ohmic dissipation

$$
\mathrm{D}_{\sigma}(\omega) \approx \iiint_{\mathrm{CV}} \sigma\left[\left(\omega_{\eta}-\omega^{*}\right)^{\prime} \mathrm{r}^{2} \sin \theta \mathrm{B}_{\mathrm{r}}-\left(\omega_{\eta}-\omega^{*}\right) \mathrm{r}_{\phi} \mathrm{B}_{\phi}\right]^{2} \mathrm{dV}
$$

If the advective term proportional to $\partial_{\phi} B_{\phi}$ were omitted, then (13) would resemble (9a) with an 'effective viscosity $\rho \chi^{\prime}$ equal to or ${ }^{2} \mathrm{~B}_{\mathrm{r}}{ }^{2}$. With $\sigma \approx 3 \times 10^{5} \mathrm{~S} / \mathrm{m}$ and $\mathrm{B}_{\mathrm{r}}{ }^{2} \approx(3.3 \text { gauss })^{2}$ averaged over $|\mathrm{r}|=\mathrm{c}, \rho \chi$ is about $4 \times 10^{11} \mathrm{~Pa}$ $s$ and $\chi$ is about $4 \times 10^{7} \mathrm{~m}^{2} / \mathrm{s}$. The latter value is about 14 orders of magnitude larger than the molecular kinematic shear viscosity of the liquid metal. This may help explain differences, highlighted by Lumb \& Aldridge (1991), between 'effective viscosities' obtained presuming a non-magnetic core and the viscosity of the liquid metal. Appendix A shows how such oversimplification of $D_{\sigma}(\omega)$ destroys boundary layer structure yet, with non-zero $\lambda$, leads to seemingly reasonable values for inner core eastward drift.

\subsection{Variational Calculus and Illustrative Solution}

With $\Delta \mathrm{L}_{\mathrm{Z}}$ from (5b) and $\mathrm{D}\left(\omega, \omega^{\prime}\right)$ equal to the sum of magnetic dissipation (12c) and viscous dissipation (9a), the stationarity condition (8) is written

$$
\delta\left\{(8 \pi / 3) \int_{d}^{c}\left[2 P_{m} \eta^{-1}\left(\omega-\omega_{B}-\omega^{*}\right)^{2}+\rho v\left(\omega^{\prime}\right)^{2}+\lambda \rho \omega\right] r^{4} d r\right\}=0
$$

The lower limit of integration has been reset to $\mathrm{d}$ for a rigidly rotating, magnetically locked inner core. Stationarity requires the integrand of $(14)$, denoted $f\left(\omega, \omega^{\prime} ; r\right)$, to satisfy Euler's equation, which sets $\partial f / \partial \omega$ equal to $\left(\partial f / \partial \omega^{\prime}\right)^{\prime}$ (see, e.g., Marion, 1970) and reduces to

$$
\omega^{\prime \prime}+\left[\ln \left(\rho v r^{4}\right)\right]^{\prime} \omega^{\prime}-2 P_{m}(\rho v \eta)^{-1}\left(\omega-\omega_{B}-\omega^{*}\right)=\lambda / 2 v
$$

To simplify (15), omit variations in $\rho, v$, and $\eta$ with radius and set $\lambda$ to zero. For such stationary dissipation,

$$
\omega^{\prime \prime}+(4 / \mathrm{r}) \omega^{\prime}-2 \mathrm{P}_{\mathrm{m}}(\rho \vee \eta)^{-1}\left(\omega-\omega_{\mathrm{B}}-\omega^{*}\right)=0 .
$$

Equation (16) also describes a balance between azimuthal Lorentz and viscous forces (see Appendix B). The general solution to (16) is the sum of particular solution $\omega_{p}$ and homogeneous solution $\xi$. Particular solution $\omega_{p}=$ $\omega_{\mathrm{B}}+\omega^{*}$ satisfies

$$
\omega_{\mathrm{p}}{ }^{\prime \prime}+(4 / \mathrm{r}) \omega_{\mathrm{p}}^{\prime}=0
$$


and is

$$
\omega_{\mathrm{p}}(\mathrm{r})=\omega_{0}+\mathrm{B}(\mathrm{c} / \mathrm{r})^{3}
$$

where $\omega_{0}$ and B are integration constants. Primary spherical Couette flow (17b) also describes a non-magnetic case (Cartwright et al., 1996; B. Bills, 1998); non-zero $\lambda$ adds a quadratic to $\omega_{\mathrm{p}}(\mathrm{r})$. Homogeneous solution $\xi$ satisfies

$$
\xi^{\prime \prime}+(4 / \mathrm{r}) \xi^{\prime}-2 \mathrm{P}_{\mathrm{m}}(\rho \vee \eta)^{-1} \xi=0 \text {. }
$$

The functional form of $\mathrm{P}_{\mathrm{m}}$ (r) is needed to solve (18a). The detailed form does not matter much for the problem at hand, provided $\mathrm{P}_{\mathrm{m}}$ (r) does not vary by more than eight orders of magnitude across the outer core, because $2 \mathrm{P}_{\mathrm{m}} \mathrm{r}^{2}(\rho \vee \eta)^{-1}$ is enormous and $\xi$ is therefore small except in thin boundary layers.

If the meridional magnetic field originates in the core, then positive $\mathrm{P}_{\mathrm{m}}(\mathrm{r})$ should tend to increases with depth below c, perhaps reaching a maximum and then falling to modest values. The illustrative case simply takes $\mathrm{P}_{\mathrm{m}}(\mathrm{r})$ to be $\mathrm{Kc}^{2} / \mathrm{r}^{2}$, so solutions to (18a) are of power law form

$$
\xi=C(r / c)^{p}+D(r / d)^{q},
$$

where

$$
\begin{aligned}
\mathrm{p} & =(1 / 2)\left\{-3+\left[9+\left(8 \mathrm{Kc}^{2} / \rho v \eta\right)\right]^{1 / 2}\right\} \\
& \approx\left(2 \mathrm{Kc}^{2} / \rho v \eta\right)^{1 / 2} \approx 2 \times 10^{7} \\
\mathrm{q} & =(1 / 2)\left\{-3-\left[9-\left(8 \mathrm{Kc}^{2} / \rho v \eta\right)\right]^{1 / 2}\right\} \approx-\mathrm{p}
\end{aligned}
$$

The value of $p$ in (18c) follows from: a 5 gauss meridional field at radius $c=3.48 \mathrm{Mm}$, hence $\mathrm{K} \approx 0.1 \mathrm{~Pa} ; \rho \approx 10^{4}$ $\mathrm{kg} / \mathrm{m}^{3}$; the molecular kinematic shear viscosity $\vee$ of the molten metal, which is well-known to be about $3 \times 10^{-7} \mathrm{~m}^{2} / \mathrm{s}$ (Poirier, 1988); and $\sigma \approx 3 \times 10^{5} \mathrm{~S} / \mathrm{m}$ as above. Because $\mathrm{p}$ is so large, the first and second terms in (18b) respectively dominate the core-mantle (outer) and inner-outer core (inner) boundary layers. The outer boundary layer scale height $\left|\xi / \xi^{\prime}\right|$ is $\delta=c / p \approx 20 \mathrm{~cm}$ (about 2.5 times the Ekman depth). The inner boundary layer scale height is $\varepsilon \delta$. If $\mathrm{P}_{\mathrm{m}}(\mathrm{r})[\rho \vee \eta]^{-1}$ were decreased by eight orders of magnitude, $\delta$ would increase to $2 \mathrm{~km}$, which remains thin compared with $2.2 \mathrm{Mm}$ of molten iron. The illustrative case is therefore robust.

The general solution of (16) is the sum of (17b) and (18b)

$$
\omega(\mathrm{r})=\omega_{0}+B(\mathrm{c} / \mathrm{r})^{3}+\mathrm{C}(\mathrm{r} / \mathrm{c})^{\mathrm{p}}+\mathrm{D}(\mathrm{r} / \mathrm{d})^{\mathrm{q}}
$$

Spherical vortex (19) exerts no net azimuthal force density in the outer core in so far as viscous diffusion of its momentum balances Lorentz drag. 


\subsection{Boundary Conditions for Magnetically Locked Inner Core}

The four parameters $\left(\omega_{0}, B, C, D\right)$ in (19) are precisely those needed to match bounday conditions (3c-f). The contribution to $\omega(\mathrm{c})$ from $D(c / d)^{q}=D(c / d)^{* *} q \approx D \varepsilon^{* *}\left(2 \times 10^{7}\right)$ proves negligibly small, so the no-slip core-mantle interface condition (3d) on (19) implies

$$
\omega_{0}=-\mathrm{B}-\mathrm{C} .
$$

Westward drift condition $(3 c)$ is applied at $c^{-} \approx c-10 \delta$. To an accuracy of $[(c-10 \delta) / c]^{p} \approx 10^{-5}$, this gives

$$
\omega\left(\mathrm{c}^{-}\right)=\omega_{0}+\mathrm{B}=-\mathrm{C} \text {. }
$$

Similarly neglecting the small contribution from $\mathrm{C}^{\mathrm{p}}$ to $\omega(\mathrm{d})$,

$$
\omega(\mathrm{d})=B\left[(\mathrm{c} / \mathrm{d})^{3}-1\right]-\mathrm{C}+\mathrm{D} .
$$

The top of the inner boundary layer is taken to be $d^{+}=d+10 \varepsilon \delta$, so

$$
\omega\left(d^{+}\right) \approx B\left[(\mathrm{c} / \mathrm{d})^{3}-1\right]-\mathrm{C} .
$$

By subtracting (20c) from (20d), we see that the jump in $\omega$ across the inner boundary layer is $-D$. Thus $|\mathrm{D}|$ is small when $\omega^{\prime}$ (d) is zero, as required by condition (3f). The latter is intended to reduce dissipation in the inner-outer core transition region that does not immediately aid core cooling. Because $\mathrm{Cp \varepsilon} \mathrm{p}_{<} \mathrm{C}$, this condition gives

$$
\begin{aligned}
\omega^{\prime}(\mathrm{d}) & =-(3 \mathrm{~B} / \mathrm{c})(\mathrm{c} / \mathrm{d})^{4}+(\mathrm{Cp} / \mathrm{c})(\mathrm{d} / \mathrm{c})^{\mathrm{p}-1}+(\mathrm{Dq} / \mathrm{d}) \\
& =(\mathrm{Dq} / \mathrm{d})-3 \mathrm{~B}(\mathrm{c} / \mathrm{d})^{4}=0
\end{aligned}
$$

hence

$$
\mathrm{D}=3 \mathrm{q}^{-1} \mathrm{~B}(\mathrm{c} / \mathrm{d})^{3} .
$$

Recalling $q \approx-2 \times 10^{7},(20 f)$ confirms $|D| \ll|B|$. Substitution of $(20 a, b, f)$ into (19) gives

$$
\omega(\mathrm{r})=\omega\left(\mathrm{c}^{-}\right)\left[1-(\mathrm{r} / \mathrm{c})^{\mathrm{p}}\right]+\mathrm{B}\left[(\mathrm{c} / \mathrm{r})^{3}-1+\left(3 / \mathrm{q}^{-}\right)(\mathrm{c} / \mathrm{d})^{3}(\mathrm{r} / \mathrm{d})^{\mathrm{q}}\right]
$$

To relate $B$ to $\omega\left(c^{-}\right)$, and thus assign a numerical value to $\omega(d)$ via $(3 c)$ and $(20 d)$, it is assumed that $\Delta L_{Z}$ in (5b) is zero. (Alternatively, $\Delta \mathrm{L}_{\mathbf{z}}$ might be calculated from a value for $\omega(\mathrm{d})$ or constrained via non-zero $\lambda$ ). With $\rho$ already treated as uniform and omitting the tiny contribution to $\Delta \mathrm{L}_{\mathrm{z}}$ from the boundary layers, this requires

$$
0=\omega(d) d^{5} / 5+\int_{d^{+}}^{c^{-}}\left\{\omega\left(c^{-}\right)+B\left[(c / r)^{3}-1\right]\right\} r^{4} d r .
$$


The elementary integration, with $\omega(d)$ being $\omega\left(c^{-}\right)+B\left[(c / d)^{3}-1\right)$ from $(20 b$, d $)$, gives

$$
\mathbf{B}=-2 \omega\left(c^{-}\right)\left[3-\varepsilon^{2}\right]^{-1} \text {. }
$$

Substitution of (22b) into (21) yields the solution

$$
\omega(\mathrm{r})=\omega\left(\mathrm{c}^{-}\right)\left\{\left[1-(\mathrm{r} / \mathrm{c})^{\mathrm{p}}\right]+2\left(3-\varepsilon^{2}\right)^{-1}\left[(\mathrm{c} / \mathrm{r})^{3}-1+(3 / \mathrm{q})(\mathrm{c} / \mathrm{d})^{3}(\mathrm{r} / \mathrm{d})^{\mathrm{q}}\right]\right\} .
$$

Clearly (22c) depends wholly upon $\omega\left(c^{-}\right)$. If $\omega\left(c^{-}\right)$were presumed to be zero, then $\omega(r)$ would vanish; if $\left|\omega\left(c^{-}\right)\right|$ were presumed to vastly exceed $\left|\omega_{B}\left(c^{-}\right)\right|$, then $\omega(r)$ would be quite large. Geomagnetic inversions indicate an intermediate case wherein $\omega\left(c^{-}\right)$is a large fraction of $\omega_{B}\left(c^{-}\right)$.

By (22c), or by combining (22b) with (20d) and (20b),

$$
\begin{aligned}
\omega(d) & =\omega\left(c^{-}\right)\left[1+\frac{2\left(\varepsilon^{3}-1\right)}{3 \varepsilon^{3}-\varepsilon^{5}}\right] \\
& =-\omega\left(c^{-}\right)[13.31]
\end{aligned}
$$

In the diffusive extreme, $\omega\left(c^{-}\right)$and $\omega(d)$ would be zero. In the frozen-flux extreme, $\omega\left(c^{-}\right)$would be $\omega_{B}$ and, using $\omega_{\mathrm{B}}$ from (3b),

$$
\omega(\mathrm{d})=-\omega_{\mathrm{B}}\left(\mathrm{c}^{-}\right)[13.31]=1.54^{\circ} / \mathrm{yr} .
$$

Equation (23c) offers a soft upper bound on inner core eastward drift.

The shear across the core-mantle boundary layer in the illustrative example is

$$
\omega^{\prime}(\mathrm{c})=\mathrm{q}^{+} \mathrm{C} / \mathrm{c}-2 \mathrm{~B} / \mathrm{c} \approx-\omega\left(\mathrm{c}^{-}\right) / \delta .
$$

The feeble viscous torque exerted by (24) on the mantle must be compensated by magnetic torques on the resistive mantle or magnetically permeable crust; however, even with the frozen-flux value for $\omega\left(c^{-}\right)$, a very weak toroidal magnetic field at the core-mantle interface, albeit of magnitude no less than $8 \mathrm{nT}$, could suffice (Voorhies, 1991). If it were not compensated, the resulting secular increase in the length of the day of roughly $5.5 \mu \mathrm{s} / \mathrm{decade}$, or less than one hour per $4.5 \mathrm{Gyr}$, would seem far smaller than other effects omitted above.

Both viscous and magnetic dissipation due soley to spherical vortex (22c) are concentrated in the core-mantle boundary layer and sum to a tiny fraction of the geothermal flux. Viscous dissipation (9a) in the core-mantle boundary layer due vortex (22c) alone would be about $\left(4 \pi c^{4} \rho v / 3 \delta\right)\left[\omega\left(c^{-}\right)\right]^{2}$, or $40 \mathrm{~kW}$. Magnetic dissipation (12c) due to this vortex alone is due solely to the homogeneous solution (18b). For the form of $\mathrm{P}_{\mathrm{m}}$ adopted above, the 
portion of this disspation occuring in the core-boundarly layer would be about $\left(4 \pi c^{4} \mathrm{~K} \mu \sigma / 3 \delta\right)\left[\omega\left(c^{-}\right)\right]^{2}$, or about 0.5 MW. These values seem very small; however, if other motions of the outer core dissipated magnetic and kinetic energy at the same rate per unit volume, then total disspation in the outer core would be about 3 TW. Dissipation of this magnitude might arise if the other motions continually entrain and mix thin boundary layers into the body of the outer core or, perhaps more simply, have a very small scale in one direction.

\section{Summary}

The idea that geomagnetic westward drift indicates a slight convective leveling of the momentum gradient within Earth's core was pursued in search for a differentially rotating mean state, upon which various oscillations and secular effects might be superimposed. The variational calculus of stationary dissipation applied to a spherical vortex within Earth's core leads to an inhomogeneous second order differential equation for the differential rotation profile $\omega(\mathrm{r})$. For a magnetic, liquid metallic outer core $\left(\mathrm{P}_{\mathrm{m}} \mathrm{r}^{2} / \rho v \eta>1\right)$, the bulk of the vortex is separated from the bounding solids by $20 \mathrm{~cm}$ thin magneto-viscous boundary layers.

Because part of the motion may induce no electric current, hence no Ohmic dissipation, four boundary conditions are required instead of two. The four conditions imposed on this form are: (i) no-slip at the core-mantle interface, (ii) geomagnetically estimated bulk westward flow of up to $0.12 \% \mathrm{yr}$ at the base of the thin core-mantle sub-layer, (iii) no-slip at the inner-outer core interface, and (iv) the hydrodynamically stress-free inner core boundary condition appropriate to a magnetically locked inner core. To compute the eastward drift of such an inner core from geomagnetic westward drift, it is assumed that the axial core angular momentum anomaly is zero.

The eastward drift of the inner core resulting from this analytic exercise is at most $1.5 \% \mathrm{yr}$. This value is within the $0-3 \% \mathrm{yr}$ range predicted by some numerical simulations and inferred from seismological data. It is remarkable that a simple, kinematic spherical vortex model featuring liquid metallic viscosity should show any similarity to either. Values temporarily exceeding $1.5 \%$ yr might be explicable in terms of decadal oscillations. Provided the axial angular momentum anomaly of the core is not too large, there appears to be no serious conflict between geomagnetically inferred westward drift at the top of the core, eastward drift at depth, and seismologically inferred eastward drift of the inner core. The general agreement by no means reduces the importance of inner-outer core-mantle oscillations, differential precession, differential tidal despin, or differential spin up due to inner core solidification. 
Acknowledgements. Special thanks to B. Bills for encouraging me to write this paper. My gratitude to the people of the United States of America who supported this research via their National Aeronautics and Space Administration (RTOP 622-72-03).

\section{APPENDIX A}

If $\partial_{\phi} \mathrm{B}_{\phi}$ were omitted and heterogeneous $\sigma \mathrm{r}^{2} \mathrm{~B}_{\mathrm{r}}{ }^{2}$ parameterized as a laterally homogeneous 'effective viscosity' $\rho(\mathbf{r}) \chi(\mathbf{r})$, then (13) would reduce to

$$
D_{\sigma} \approx(8 \pi / 3) \int_{0}^{c} \rho \chi r^{4}\left(\omega^{\prime}\right)^{2} d r .
$$

The similarity between (A1) and (9a) provokes comment on dynamic shear viscosity $\rho v$ as a parameterization of momentum transport by electromagnetic molecular interactions ("collisions"). Hydrodynamic stress $\left(\mathrm{P}_{\mathrm{ij}}=-\mathrm{p} \delta_{\mathrm{ij}}+\right.$ $\mathrm{p}_{\mathrm{ij}}$ ) may be viewed as a macroscopic average over intermolecular electromagnetic (Maxwell) stress $\left\langle\mathrm{T}_{\mathrm{ij}}\right\rangle$. The reference stress $\mathrm{T}_{\mathrm{ij}}^{\mathrm{o}}$ is due to a tangle of fluctuating intermolecular fields (e, b) originating in the molecules. A macroscopic average (e.g., over a cubic millimeter and a milli-second) amounts to an isotropic cohesive equilibrium $\left\langle\mathrm{T}_{\mathrm{ij}} \mathrm{O}^{\mathrm{O}}=-\mathrm{p} \delta_{\mathrm{ij}}\right.$. Applied macroscopic strain rate $\omega^{\prime} \mathrm{r} \sin \theta$ shears the intermolecular field within a liquid, arguably inducing a contribution to $b_{\phi}$ equal to $\omega^{\prime} \operatorname{rsin} \theta \kappa b_{r}$, where $\kappa$ depends upon details of the interactions. The contribution to $<\mathrm{T}_{\mathrm{r} \phi}>$ is $\left\langle\mathrm{b}_{\mathrm{r}} \mathrm{b}_{\phi}>/ 2 \mu_{0}\right.$, or $\left(\sin \theta / 2 \mu_{\mathrm{O}}\right)<\kappa b_{\mathrm{r}}{ }^{2}>\omega^{\prime}$. In the absence of macroscopic fields, $<\mathrm{b}>$ remains zero; moreover, terms analogous to those omitted in going from (13) to (A1), specifically $\left\langle\left(\partial_{\phi} b_{\phi}\right)^{2}>\right.$ and $\left\langle b_{\mathbf{r}} \partial_{\phi} b_{\phi}\right\rangle$, would seem as negligible as $\left\langle\partial_{\phi} \mathrm{b}_{\phi}\right\rangle$ when the applied strain rate is uniform over the averaging 4-volume. If the contribution to $\left\langle\mathrm{T}_{\mathrm{r} \phi}>\right.$ is identified with $\mathrm{p}_{\mathrm{ij}}$, then $\rho v$ might be identified with $\left\langle\kappa \mathrm{b}_{\mathrm{r}}{ }^{2}>/ 4 \mu_{\mathrm{O}}\right.$. The fact remains that interactions establishing viscosity involve intermolecular collisions, while macroscopic $\mathbf{B}$ is due to Amperian currents regulated by conduction electron collisions; therefore, $\kappa$ differs from $\sigma$ and $v$ differs from $\chi$. Large values of core 'effective viscosity' have little or nothing to do with viscosity, but may have much to do with Lorentz forces and Ohmic dissipation.

If $\mathrm{D}(\omega)$ were treated as the sum of $(\mathrm{A} 1)$ and $(9 \mathrm{a})$, then (8) would amount to

$$
0 \approx \delta(8 \pi / 3) \int_{0}^{c}\left[\rho \chi\left(\omega^{\prime}\right)^{2}+\lambda \rho_{0}(r) \omega(r)\right] r^{4} d r
$$

because $v<<\chi$. Stationarity would require the integrand of (A2) to satisfy Euler's equation, 


$$
\omega^{\prime \prime}+\left[\ln \left(\rho \chi r^{4}\right)\right]^{\prime} \omega^{\prime}-\lambda / 2 \chi=0 .
$$

The first and second integrals of (A3) are straightforward when $\rho(\mathrm{r})$ and $\chi(\mathrm{r})$ are, respectively, approximated by uniform constants. The result is written

$$
\omega(r)=\omega_{1}-\left(C_{1} / 3 \rho \chi\right) r^{-3}+(\lambda / 20 \chi) r^{2}
$$

where $C_{1}$ and $\omega_{1}$ are the two constants of the two integrations. In (A4), primary spherical Couette flow is the homogeneous solution and the parabolic term on the far right is the particular solution for non-zero $\lambda$. The constrast with (19) is remarkable; moreover, (A4) shows no sign of the boundary layers and, even with non-zero $\lambda$, can sate but three of four boundary conditions.

Stress Free CMB vs. ICB: If $\omega^{\prime}\left(c^{-}\right)$were zero, then (A4) would imply

$$
(\lambda / \chi)=-10\left(C_{1} / \rho \chi\right) c^{-5}
$$

When (A4, A5a) are used in (5b) and $\Delta \mathrm{L}_{\mathrm{z}}$ is set to zero, again for uniform density, one obtains

$$
\left(\mathrm{C}_{1} / \rho \chi\right)=\omega_{1} \mathrm{c}^{3} / 5 \mathrm{Q},
$$

where $\mathrm{Q}$ depends only on $\varepsilon$ and is about 0.2259 . It has also been shown that

$$
\omega_{1}=\omega\left(c^{-}\right) 6 \mathrm{Q} /(6 \mathrm{Q}-1) .
$$

Subsitution of (A5a-c) into (A4) and evaluation of the result at inner radius d yields

$$
\begin{aligned}
\omega(\mathrm{d}) & =\omega\left(\mathrm{c}^{-}\right)[6 \mathrm{Q} /(6 \mathrm{Q}-1)]\left\{1-(5 \mathrm{Q})^{-1}\left[\left(3 \varepsilon^{3}\right)^{-1}+\varepsilon^{2} / 2\right]\right\} \\
& =-\omega\left(\mathrm{c}^{-}\right)(22.68)=2.62^{\circ} / \mathrm{yr},
\end{aligned}
$$

where the last step uses the frozen-flux value for $\omega\left(c^{-}\right)$from (3c). One also obtains an $\omega_{1}$ of $-2.44 \times 10^{-10} \mathrm{rad} \mathrm{s}^{-1}$ and $C / \rho \chi=-9.1 \times 10^{9} \mathrm{~m}^{3} / \mathrm{s}$.

The foregoing oversimplification of $D_{\sigma}(\omega)$ leaves some shear at the inner core boundary; indeed, from (A4 A5d) it follows that

$$
\begin{aligned}
\omega^{\prime}(d) & =\omega(c) c^{-1}(6 / 5)(6 Q-1)^{-1}\left[3 \varepsilon^{-4}-\varepsilon\right] \\
& =224.2 c^{-1} \omega\left(c^{-}\right)=-28.3 \omega(d) / d .
\end{aligned}
$$

Such shear could curl field lines threading the inner core and generate non-irrotational currents, magnetic diffusion, Ohmic dissipation that might inhibit inner core solidification, and restoring Lorentz torques. If it were not 
compensated by other torques, the viscous stress would tend to spin down the inner core and spin up the outer core, albeit extremely slowly.

If the hydrodynamic stress-free condition were instead applied at the inner boundary, then a slower inner core eastward drift of $-1.847 \omega(\mathrm{c})$, or about $0.21 \% \mathrm{yr}$, would result. The non-zero shear on the main-stream below the core-mantle boundary, $\omega^{\prime}\left(c^{-}\right)$, would be about be about $7.08 \omega\left(c^{-}\right) / c$. If uncompensated, this would tend to spin up the core and spin down the mantle.

\section{APPENDIX B: Force Balances}

The azimuthal Lorentz force density implied by $(10 a-e)$ is $\mathrm{J}_{r}(\omega) \mathrm{B}_{\theta}-\mathrm{J}_{\theta}(\omega) \mathrm{B}_{\mathrm{r}}$, is $-\sigma\left(\omega-\omega_{\mathrm{B}}{ }^{\omega}{ }^{*}\right) \mathrm{r} \sin \theta\left(\mathrm{B}_{\mathrm{r}}{ }^{2}+\right.$ $\left.\mathrm{B}_{\theta}{ }^{2}\right)$, and is equivalent to magnetic friction $-\mathrm{C}_{f}\left(\mathrm{~V}_{\phi}-\mathrm{V}_{\mathrm{B}}-\mathrm{V}^{*}\right)$ with drag coefficient $\mathrm{C}_{f} \equiv \sigma\left(\mathrm{B}_{\mathrm{r}}^{2}+\mathrm{B}_{\theta}^{2}\right)$. The azimuthal viscous force density from the divergence of $\mathrm{p}_{\mathrm{r} \phi}$ is, assuming uniform $\rho v, \rho v \sin \theta\left(\mathrm{r} \omega^{\prime}{ }^{\prime}+4 \omega^{\prime}\right)$, or $\rho v r^{-3}\left(r^{4} \omega^{\prime}\right)^{\prime}$. There is no azimuthal Coriolis force on motions $v_{\phi}$; pressure forces are scaloidal; and buoyancy forces are mainly radial. A balance between Lorentz drag and viscous diffusion would have

$$
\rho v \sin \theta\left(\mathrm{r} \omega^{\prime \prime}+4 \omega^{\prime}\right)-\sigma\left(\omega-\omega_{\mathrm{B}}-\omega^{*}\right) \mathrm{r} \sin \theta<\mathrm{B}_{\mathrm{r}}^{2}+\mathrm{B}_{\theta}{ }^{2}>=0 \text { : }
$$

Only the azimuthal mean value of $\left(\mathrm{B}_{\mathrm{r}}{ }^{2}+\mathrm{B}_{\theta}{ }^{2}\right)$, denoted by angle brackets, appears in (B1) because other currents $\mathbf{J}$ $\mathbf{J}(\omega)$, and other motions $\mathbf{v}-\mathbf{V}(\omega)$, in the core are excluded from the analysis of the vortex alone. When (B1) is multiplied by $\sin ^{2} \theta d \theta d \phi$ and integrated over a sphere, the result is identical to (16). Spherical vortex (19) is thus 'force-free' in the limited sense that the viscous diffusion of its momentum is balanced by the Lorentz force caused by the current it generates from the ambient field.

\section{REFERENCES}

Aurnou, J.M., Brito, D., and Olson, P., 1996. Mechanics of inner core super-rotation. Geophys. Res. Lett., 23: 3401-3404.

Aurnou, J., Brito, D., and Olson, P., 1998. Anomalous rotation of the inner core and the toroidal magnetic field. J. Geophys. Res., 103: 9721-9738.

Bills, B.G., 1998. Tidal despinning of the mantle, inner core super-rotation and outer core effective viscosity. Submitted to J. Geophys. Res..

Buffett, B.A., 1997. Geodynamic estimates of the viscosity of the Earth's inner core. Nature, 388: 571-573.

Cartwright, J.H.E., Feingold, M., and Piro, O., 1996. Chaotic advection in three dimensional unsteady incompressible laminar flow. J. Fluid Mech., 316: 259-284.

Chandrasekhar, S., 1961. Hydrodynamic and Hydromagnetic Stability. Oxford University Press; Dover edition, Toronto. $652 \mathrm{pp}$. 
Creager, K., 1997. Inner core rotation from small scale heterogeneity and time-varying travel times. Science, 278: 1284-1288.

Glatzmaier, G.A., and Roberts, P.H., 1995a. A three-dimensional self-consistent computer simulation of a geomagnetic field reversal. Nature, 377: 203-205.

Glatzmaier, G.A., and Roberts, P.H., 1995b. A three-dimensional convective dynamo solution with rotating and finitely conducting inner core and mantle. Phys. Earth Planet Int., 91: 63-75.

Glatzmaier, G.A., and Roberts, P.H., 1996. Rotation and magnetism of Earth's inner core. Science, 274: 18871991.

Gubbins, D., 1981. Rotation of the inner core. J. Geophys. Res., 86: 11695-11699.

Gubbins, D., and Roberts, P.H., 1987. Magnetohydrodynamics of the Earth's core. In Geomagnetism, Vol. 2, J.A. Jacobs, ed.. Academic Press, New York. 579 pp.

Halley, E., 1692. An account of the cause of the change of the variation of the magnetic needle; with an hypothesis of the structure of the internal parts of the earth. Phil. Trans. Roy. Soc. Lond., XII: 208-221.

Kennet, B.L., Engdahl, E.R., and Buland, R., 1995. Constraints on seismic velocities in the Earth from traveltimes. Geopys. J. Int:: 108-124.

Lumb, L.I., and Aldridge, K.D., 1991. On viscosity estimates for the Earth's fluid outer core and core-mantle coupling. J. Geomag. Geoelectr., 43: 93-110.

Marion, J.B., 1970. Classical Dynamics. Academic Press, New York. 573 pp.

Poirer, J.P., 1988. Transport properties of liquid metals and the viscosity of Earth's core. Geophys. Atrophys. Fluid Dyn., 9: 99-105.

Song, X. and P. G. Richards, 1996. Seismological evidence for differential rotation of the Earth's inner core. Nature, 382: 221-224.

Souriau, A., Roundil, P., and Moynot, B., 1997. Inner core differential rotation: facts and artefacts. Geophys. Res. Lett., 24: 2103-2106.

Souriau, A., 1998. Is the rotation real? Science, 281: 55-56.

Su, W.-J., Dziewonski, A.M., and Jeanloz, R., 1996. Planet within a planet: rotation of the inner core of the Earth. Science, 274: 1883-1887.

Vanyo, J. P., 1984. Earth core motions: experiments with spheroids. Geophys. J. R. astr. Soc., 77: 173-183.

Vanyo, J.P., 1991. A geodynamo powered by luni-solar precession. Geophys. Astrophys. Fluid Dyn., 59: 209-234.

Voorhies, C. V., 1991. Coupling an inviscid core to an electrically insulating mantle. J. Geomag. Geoelectr., 42: 131-156.

Voorhies, C.V., 1993. Geomagnetic estimates of steady surficial core flow and flux diffusion: unexpected geodynamo experiments. In Dynamics of Earth's Deep Interior and Earth Rotation, J.-L. LeMouel, D.E. Smylie, and T. Herring, eds.. Geophys. Monogr. Ser., 72: 113-125.

Voorhies, C.V., 1995. Time-varying fluid flow at the top of Earth's core from Definitive Geomagnetic Reference Field models. J. Geophys. Res., 100: 10029-10039. 